PRAXIS

ecucativa

Universidad Nacional de La Pampa

Facultad de Ciencias Humanas

Instituto de Ciencias de la Educación

para la investigación interdisciplinaria

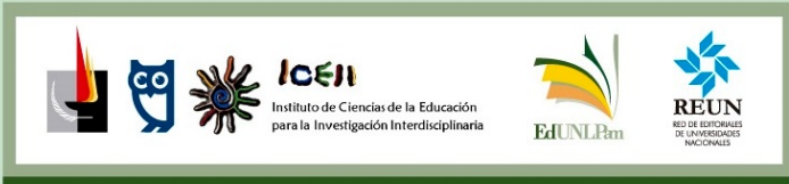

ISSN 2313-934X

SANTA ROSA, LA PAMPA, ARGENTINA

Correo electrónico: iceii@humanas.unlpam.edu.ar

Disponible en https://cerac.unlpam.edu.ar/index.php/praxis

Mis ideas en tres minutos: futuros maestros que escriben y reflexionan. Artículo de Adriana Inés Ávila Zárate y Allan Amador Díaz Rueda. Praxis educativa, Vol. 25, No 3 septiembre - diciembre 2021. E - ISSN 2313-934X. pp. 1-19. https://dx.doi.org/10.19137/praxiseducativa2021-250303

Esta obra se publica baja Licencia Creative Commons 4.0 Internacional CC BY- NC-SA Atribución, No Comercial, Compartir igual

(c) (†) $\$$

\title{
Mis ideas en tres minutos: futuros maestros que escriben y reflexionan
}

My ideas in three minutes: future teachers who write and reflect

Minhas ideias em três minutos: futuros professores que escrevem e refletem

\section{Adriana Inés Ávila Zárate}

Universidad Autónoma de Bucaramanga, Colombia

aavila2@unab.edu.co

ORCID: 0000-0002-4122-7960

\section{Allan Amador Díaz Rueda}

Universidad Autónoma de Bucaramanga, Colombia

adiaz95@unab.edu.co

ORCID 0000-0002-4433-5633

Recibido: 2021- 02-12 | Revisado: 2021-04-03 | Aceptado: 2021-05- 07 


\section{Resumen}

El presente artículo reporta los resultados de la sistematización de la experiencia Mis ideas en tres minutos, actividad implementada en el curso Escuela Maternal de la Licenciatura en Educación Infantil en la Universidad Autónoma de Bucaramanga, Colombia. Se empleó la técnica One minute paper para favorecer la reflexión de los estudiantes sobre su futuro rol como maestros, mediante la composición de un texto que contuviera el análisis de sus experiencias y prácticas. Se hizo acopio de esos escritos y se usó el programa Nvivo para analizar los datos a partir de las siguientes categorías: identidad como maestros y apropiación conceptual. Sin embargo, durante el trabajo, surgieron categorías emergentes, como apreciaciones sobre el sistema educativo y otras relacionadas con la escritura. Las conclusiones señalan el potencial que posee esta técnica para que se realice de manera ágil un ejercicio reflexivo, al tiempo que genera en los estudiantes el hábito de cuestionarse constantemente, posibilitando que sean cada vez más autocríticos.

Palabras clave: práctica pedagógica; identidad; formación inicial.

\section{Abstract}

This article reports the results of the systematization of the experience named My ideas in three minutes, an activity implemented in the Maternal School course of the Bachelor's Degree in Early Childhood Education at Universidad Autónoma de Bucaramanga, Colombia. The One minute paper technique was used to encourage students to reflect on their future role as teachers, by composing texts containing the analysis of their experiences and practices. Those documents were collected and analyzed, using Nvivo program, based on the following categories: identity as teachers and conceptual appropriation. However, during the process, some categories emerged, such as, appreciations about the educational system and others related to writing. The conclusions point out the potential of this technique for a reflexive exercise to be carried out in an agile way; besides, it promotes in the students the habit of constantly questioning themselves, making it possible for them to be more self-critical.

Keywords: teaching practice; identity; initial training.

\section{Resumo}

O presente trabalho reporta os resultados da sistematização da experiência Minhas ideias em três minutos, atividade implementada no curso Escola maternal da Licenciatura em Educação Infantil da Universidade Autônoma de Bucaramanga, na Colômbia. Utilizou-se a técnica One minute paper para favorecer a reflexão dos estudantes sobre o futuro papel como professores, a través de uma composição textual que tivesse a análise de suas experiências e práticas. Recolheram-se esses escritos e utilizou-se i programa Nvivo para analisar os dados a partir das seguintes categorias: Identidade como professores e apropriação conceitual. Porém, durante o trabalho surgiram categorias emergentes, como apreciações sobre o sistema educativo e outras relacionadas com a escrita. As conclusões demostram o potencial que possui essa técnica para se realizar de maneira ágil uma atividade reflexiva, ao tempo que gera nos estudantes o habito contínuo de questionar-se, possibilitando que sejam cada vez mais autocríticos.

Palavras-chave: prática pedagógica; identidade; formação inicial. 


\section{Introducción}

El presente artículo describe la sistematización de una experiencia realizada con estudiantes de quinto semestre de la Licenciatura en Educación Infantil de la Universidad Autónoma de Bucaramanga (Colombia), en el marco de la Práctica Pedagógica Escuela Maternal. El grupo de estudio estuvo conformado, de manera directa, por quince maestros en formación y, de manera indirecta, por más de 450 niños con edades entre seis meses y dos años, quienes se vieron beneficiados en sus procesos de atención y desarrollo como consecuencia de la implementación de las prácticas pedagógicas de quienes se están preparando para ser maestros de educación infantil. De forma implícita, este trabajo también involucró a los quince profesores titulares de los colegios y sus correspondientes auxiliares de los cursos en los cuales los maestros en formación UNAB realizaban sus prácticas.

El curso Práctica Pedagógica Escuela Maternal está constituido por dos momentos: el primero es la interacción directa que los practicantes realizan dos días cada semana en diferentes instituciones educativas de la ciudad, donde trabajan de manera conjunta con profesores titulares, auxiliares y niños de seis meses a dos años; el segundo es un encuentro académico, también semanal, de cuatro horas, denominado Seminario de práctica, desde el cual se realiza un trabajo teórico y analítico en el que participan los maestros en formación y la maestra responsable de dicho seminario; fue allí donde tuvo lugar la experiencia descrita.

Se partió de las preguntas: ¿cómo promover la reflexión de los estudiantes sobre su identidad docente? y ¿cómo evidenciar la apropiación conceptual del sustento teórico referente a la educación infantil? Se quiso dar prioridad al elemento reflexivo del quehacer profesoral, para articular teorías, experiencias, presaberes, talentos, etc. Para esto, se consideró la producción de textos breves, pues los futuros profesores tienen tantos compromisos académicos que no disponen de mucho tiempo para cavilar sobre sus procesos formativos. Al respecto, instrumentos como el diario pedagógico no llenan esta expectativa, debido a que se centran en la argumentación teórica; por ende, valoran más el ejercicio de teoría-práctica que las emociones y experiencias de los estudiantes. En consecuencia, los aprendices se cohíben al momento de expresar sus ideas, sin mencionar que este género discursivo usualmente es asumido como una tarea debido a que es calificable. Este factor hace que se convierta en un registro frío y esquemático.

Con base en las anteriores consideraciones, se optó por usar la técnica One minute paper. Iniciativa popularizada por Cross y Angelo (1998) que consiste en una breve actividad de valoración de la clase en la que los estudiantes retroalimentan sus procesos formativos y las dinámicas de las sesiones mediante la elaboración de un texto espontáneo que escriben en los últimos minutos de cada encuentro académico.

En el caso del curso Práctica Pedagógica Escuela maternal, los maestros en formación escribían, en los tres minutos finales de la clase, un texto a mano, con el objetivo de plasmar las ideas que registraron durante cada encuentro (reflexiones, pensamientos, sensaciones y aprendizajes). Las orientaciones dadas por la maestra asesora consistían en que el texto escrito superara el plano descriptivo y articulara las ideas, es decir, no se permitían ideas sueltas o a manera 
de listado. Cabe señalar también que este ejercicio no era calificable y que no se les brindó instrucciones complementarias ni estructura predeterminada, pues se esperaba que los estudiantes pudieran expresarse libremente.

Luego, la profesora del curso hacía retroalimentación personalizada de los textos y los devolvía a los estudiantes cada semana. En varias ocasiones, hizo un listado de los errores más frecuentes para que los aprendices los evitaran en aras de mejorar sus próximos escritos. Acto seguido, se procedió a recopilar las producciones con el fin de sistematizar la experiencia siguiendo la propuesta de Jara (2018) y se usó el programa Nvivo para el análisis de la información. Aquí, es necesario mencionar que inicialmente se definieron solo dos categorías: identidad docente y apropiación conceptual. No obstante, durante el proceso, surgieron categorías emergentes como las apreciaciones del sistema educativo que incluyen referencias a la clase, al programa de licenciatura y a los imaginarios sobre la educación infantil. Así mismo, se consideró importante incluir la categoría aspectos relacionados con los desempeños de los estudiantes en escritura; por ejemplo: la estructura de los textos (título, idea principal, desarrollo del planteamiento e idea de cierre) y la función comunicativa de las producciones (descriptivo, reflexivo, mixto, etc.).

\section{Marco referencial}

\section{One Minute Paper, base para "Mis ideas en tres minutos"}

La técnica One minute paper, de acuerdo con Vivel-Bua et al. (2015), suele vincularse al profesor Charles Schwartz de la Universidad de Berkeley y a los Harvard Assessment Seminaries, organizados con el propósito de mejorar el ejercicio docente para que el alumno tuviese un aprendizaje adecuado.

El One minute paper consiste en que, al final de la sesión de clase, el docente plantea una serie de preguntas a los alumnos orientadas a identificar conceptos clave, construir ejemplos, proponer acciones de mejora, redactar ideas y opiniones, las cuales deben ser contestadas allí mismo, de manera rápida y concreta. Luego, el profesor revisa y analiza las respuestas para identificar los errores o vacíos más frecuentes que denotan debilidades en el aprendizaje, así como las percepciones de los estudiantes con quienes se comparte el ejercicio para debatir y socializar al respecto.

Según Cuseo (2010, citado por Vivel-Bua et al., 2015), se proponen una tipología de preguntas que recogen la percepción del alumno sobre lo que le ha resultado más interesante, lo que considera más relevante o útil, su análisis del eje central de la clase y el establecimiento de conexiones conceptuales o vínculos entre los contenidos del curso. Este ejercicio le permite al profesor conocer de manera directa y a tiempo lo que están pensando, sintiendo y aprendiendo sus estudiantes, lo cual se convierte en un punto de partida muy apropiado para analizar la propia práctica pedagógica y realizar ajustes en ella.

\section{Identidad docente}


La construcción de identidad, según Beijaard et al. (2004), es un proceso relevante para la formación docente porque ayuda a comprender a los sujetos en formación y a identificar los apoyos que necesitan en su aprendizaje; implica un proceso de interacción complejo que se forma desde las personas y en interacción con otros en diferentes contextos. Por ello, tal como lo afirman Cantón y Tardif (2018), este proceso "se conjuga estrictamente con los sistemas sociales y escolares en los que obran los profesores (...), tienen identidades diversas, porque éstas están marcadas por su cultura, su lengua, las tradiciones escolares propias a su país, etc." (p. 6).

La identidad docente implica "verse a sí mismo como una determinada clase de persona y ser reconocida como tal dentro de un contexto específico, se ve afectada por los valores, por las motivaciones, las actitudes, los sentimientos y los estereotipos" (Cantón y Tardif, 2018, p. 46). Este proceso, asumido como un constructo, se va desarrollando desde que el futuro docente es estudiante y va evolucionando de acuerdo con las expectativas que tiene respecto al tipo de profesional docente que quiere ser, a los conocimientos que va apropiando y a las interacciones que vivencia.

\section{Metalenguaje}

En el artículo Una historia conceptual de los metalenguajes, González de Requena Farré, de la Universidad Austral de Chile (2019), evoca los aportes sobre la lingüística sistémico-funcional de Halliday y Matthiessen (1999) para explicar que el metalenguaje es un lenguaje que se emplea en la comprensión de otro lenguaje y se sirve de los recursos representacionales en las construcciones teóricas de diversos estratos. Esta concepción es útil para justificar por qué los futuros maestros requieren de apropiación conceptual y terminología propia de la educación inicial. De manera particular, Mis ideas en tres minutos siempre tuvo el propósito de contribuir a la comprensión de aportes teóricos sobre crianza, vínculos, prácticas del cuidado y el desarrollo del niño de cero a dos años, conceptos propios del curso Escuela Maternal.

\section{Sobre la práctica pedagógica}

El Ministerio de Educación Nacional de Colombia (MEN) define las prácticas pedagógicas como un escenario que propicia la indagación como "base para la toma de decisiones, la proyección de experiencias y estrategias pedagógicas" (MEN, 2017, p. 108), así como el deseo de explorar, conocer, comprender y preguntar. Se puede decir que la práctica es por sí misma un laboratorio en potencia para aprender a ser maestro y reflexionar al respecto. De igual manera, la concibe como "un proceso de auto reflexión, que se convierte en el espacio de conceptualización, investigación y experimentación didáctica, donde el estudiante de licenciatura aborda saberes de manera articulada que enriquecen la comprensión del proceso educativo y de la función docente" (MEN, 2014). Dado esto, se asume como un ambiente de aprendizaje para el docente en formación donde se ha de promover el desarrollo integral de sus competencias para ser maestro.

Tal definición corresponde con lo descrito por Gairín et al. (2019) cuando definen la práctica, precisamente, como "un espacio para el aprendizaje, la construcción de la identidad profesional y 
el ejercicio pre-profesional" (p. 19) que, además, favorece la actualización disciplinar de los profesores, generando un impacto en las instituciones. Los autores exponen cómo, si bien la práctica es un espacio formativo centrado en el desarrollo de competencias aplicadas, también posibilita a los estudiantes "descubrir habilidades y competencias innatas, configurar su identidad profesional y filosofía docente, reflexionar sobre el tipo de maestro que quieren ser y ampliar conocimientos" (p. 30).

Alineado a lo anterior, la Práctica Pedagógica es considerada, dentro del Programa de Licenciatura en Educación Infantil de la UNAB, como una oportunidad "donde el estudiante practicante pone a prueba en un escenario real, las competencias investigativas, pedagógicas, tecnológicas, comunicativas, ciudadanas y creativas, construidas en su proceso de formación (...) permite a los estudiantes contrastar el conocimiento adquirido con las exigencias del contexto" (Programa de Licenciatura en Educación Infantil-UNAB, 2018, p. 4). En este sentido, se asume la práctica como una actividad de carácter formativo que se desarrolla en todos los semestres para integrar la teoría y la práctica, por tanto, se:

Logra una mejor compresión del ejercicio profesional, dándole un nuevo sentido y resignificación a la realidad social, de esta manera se ofrece la oportunidad de acercar al estudiante a los posibles ámbitos en los que se desempeñará como licenciado en Educación Infantil. (Programa de Licenciatura en Educación Infantil-UNAB, 2018, p. 4)

\section{Práctica Pedagógica Escuela Maternal}

Este espacio busca que el maestro en formación identifique, comprenda y reflexione respecto a cómo las acciones de cuidado y crianza tienen estrecha relación con las acciones pedagógicas e influyen en el desarrollo de niños y niñas de cero a dos años. Se busca que el estudiante diseñe ambientes significativos de aprendizaje teniendo en cuenta elementos teóricoprácticos y del contexto que le permitan entender cómo el cuidado adecuado y el desarrollo de la primera infancia forman parte integral de la educación y es prioridad generar acciones que los favorezcan.

El concepto de cuidado integra actividades y vínculos sociales que se articulan en un complejo sistema de reciprocidad imprescindible para la supervivencia, al plantear el cuidado como una ética, al mismo tiempo que una práctica social, trasciende el ámbito de lo privado y se adentra en lo público. Desde la relación maternal como matriz de la noción de cuidado, se extiende a otras necesidades y formas de satisfacerlas, más allá del vínculo con la madre (Tobio et al., 2010, p. 2223).

Sobre la crianza, se asume como "las representaciones sociales del niño, que condicionan la interpretación de los diferentes órdenes normativos, que pueden asumir formas bastante restrictivas o muy tolerantes, dándose entre estas una variedad, que depende de los rasgos culturales del grupo" (Bocanegra, 2007, p. 5). Por tanto, reconocer dichas representaciones en los diferentes escenarios de práctica es fundamental para el licenciado en formación. 


\section{Educación Infantil}

De acuerdo con la Conferencia Mundial sobre Educación para Todos (UNESCO, 1992), a la primera infancia se le debe dar el reconocimiento que requiere, puesto que desde que el niño nace comienza a relacionarse con el mundo que lo rodea, a percibirlo; esto quiere decir que inicia un proceso de aprendizaje del entorno, donde sus experiencias influyen directamente en su desarrollo integral. La forma como interiorice sus diferentes vivencias estructura su personalidad y formas de relacionarse con el mundo. En consecuencia, los adultos deben proporcionarle los ambientes significativos para que su desarrollo sea armónico, involucrando a la familia y la escuela.

Se espera que los niños que asisten a la escuela cuenten con apoyo para el desarrollo de procesos de pensamiento, el fortalecimiento de las dimensiones del desarrollo humano, la adquisición e interiorización de aprendizajes con sentido, de modo que estén mejor preparados física y mentalmente para que su desempeño en la siguiente etapa escolar se dé naturalmente, según su edad, habilidades y contexto.

\section{Consideraciones sobre escritura académica}

Como se comentó en la introducción, durante el desarrollo de la experiencia, se identificaron algunas situaciones relacionadas con las cuatro principales dificultades de la enseñanza del proceso de escritura académica en la universidad, planteadas por Paula Carlino (2004), a saber:

1. La dificultad de los estudiantes para escribir teniendo en cuenta la perspectiva del lector

2. El desaprovechamiento del potencial epistémico de la escritura

3. La propensión de los maestros a revisar los textos solo en forma lineal y centrándose en aspectos locales y poco sustantivos

4. La dilación o postergación del momento de empezar a escribir por parte de los aprendices.

El ejercicio Mis ideas en tres minutos quiso también mostrar a los futuros maestros que el proceso de escritura puede surgir de manera espontánea, siempre y cuando se acuñe como hábito. De cierto modo, la presión por el tiempo hacía que plasmaran sus reflexiones sin tener que enfrentarse al pánico que produce "la hoja en blanco"; además, gracias a la instrucción de la maestra, los participantes pudieron reconocer el público objetivo al que debían dirigirse: los integrantes del curso Práctica Pedagógica Escuela Maternal. De manera complementaria, la retroalimentación realizada por la profesora consideró un acompañamiento global del proceso de escritura, en tanto que destacaba los aspectos positivos de las ideas planteadas por los estudiantes, cuestionaba los vacíos conceptuales, formulaba preguntas que les hiciera ahondar en las reflexiones y proponía variantes para mejorar la redacción de los textos.

Así mismo, para aprovechar el potencial epistémico de la escritura en la formación de este grupo de próximos licenciados, se consideraron los aportes sobre cómo formar profesionales reflexivos realizados por Donald Schön (1992). Según este filósofo norteamericano, la educación superior debe enfocarse en: a) elaborar un conjunto de conceptos que permitan repensar y debatir el conocimiento profesional (iniciativa de la que se desprenden términos como: práctica reflexiva, 
reflexión en la acción y conocimiento sobre la acción); b) estudiar cómo se aprende por medio de la reflexión que hacen los propios profesionales en y desde sus actividades; y c) ofrecer modelos de formación que los aprendices, maestros y futuros profesionales puedan seguir y emular sin dificultades.

\section{Método}

El presente estudio está enmarcado en el paradigma sociocrítico, específicamente se trata de una investigación cualitativa llevada a cabo mediante la metodología de sistematización de experiencias. Para Óscar Jara (2018), es un ejercicio premeditado que pretende explicar un fenómeno y reconfigurar sus saberes a través del registro, análisis y teorización de lo vivido. Para lograrlo, es necesario generar un distanciamiento de lo ocurrido que permita trascender la observación de las vivencias, sentimientos y pensamientos que produjo la intervención educativa. De este modo, "se estudia la experiencia para encontrar sus vínculos con otras prácticas sociales de las que ella forma parte" (p. 55). Con base en las ideas de dicho autor, se recorrió el siguiente camino:

1. El punto de partida: la experiencia. Haber participado en la experiencia: uno de los autores orientó el curso de Práctica Pedagógica Escuela Maternal y el otro sirvió como agente externo para registrar el desarrollo de la propuesta. Contar con registros de la experiencia: semana a semana, se recogía el ejercicio Mis ideas en tres minutos y se hacía retroalimentación personalizada a los estudiantes. También hubo dos ocasiones de retroalimentación grupal.

2. Formular un plan de sistematización. ¿Para qué queremos sistematizar? El propósito principal fue determinar la incidencia de la actividad Mis ideas en tres minutos en la reflexión de los estudiantes sobre su identidad docente y la apropiación conceptual del sustento teórico referente a la educación infantil en el curso de Práctica Pedagógica Escuela Maternal. ¿Qué experiencia sistematizamos? En este caso, se sistematizó la actividad Mis ideas en tres minutos. ¿Qué aspectos centrales nos interesan más? Dada la enorme presencia de aspectos reflexivos en los textos, el análisis se centró en las cavilaciones sobre la autopercepción de los estudiantes como maestros, su apropiación conceptual de los términos fundamentales de la práctica pedagógica en escuela maternal y sus apreciaciones sobre el sistema educativo. ¿Qué fuentes de información fueron usadas? Se contó con 150 textos que escribieron los estudiantes para plasmar las ideas que registraron durante las sesiones de clase (reflexiones, pensamientos, sensaciones). Así mismo, se implementó un formato de autoevaluación de los escritos que era diligenciado por los aprendices después de recibir la retroalimentación. ¿Qué procedimiento concreto se siguió y en qué tiempo? El ejercicio fue aplicado en el segundo semestre académico de 2019 y tuvo una duración de dieciséis semanas. La profesora usaba un proceso cíclico en el que recogía el ejercicio, hacía retroalimentación personalizada y propiciaba el nuevo espacio de escritura reflexiva. También hubo dos ocasiones de retroalimentación grupal. Debido a que las producciones fueron elaboradas a mano, fue necesario transcribirlas en versión digital. Posteriormente, 
se procedió a realizar el análisis con programa Nvivo, lo que permitió dar cuenta de los resultados de la experiencia. Con base en ellos, se elaboraron las conclusiones que serán presentadas al final de este documento.

3. La recuperación del proceso vivido. Para tal efecto, se tuvo en cuenta, además de los textos elaborados por los estudiantes, las anotaciones de la maestra en su diario de campo y las observaciones periódicas que el docente acompañante realizaba luego de escuchar cada avance del proceso. Esto se realizó en la sección "Memorandos" del programa Nvivo.

4. Procesos de análisis, síntesis e interrelaciones. Se usó el software Nvivo para hacer un análisis axial de los textos, de ello se obtuvo un gráfico de frecuencia de palabras con la intención de tener una percepción global de las reflexiones que escribían los estudiantes. Del mismo modo, se graficaron todos los títulos de los textos para conocer las inclinaciones o tendencias. Más adelante, los investigadores leyeron todos los escritos, codificaron los hallazgos y evaluaron los desempeños de los estudiantes en cada categoría. De igual modo, seleccionaron los fragmentos más dicientes para ilustrar los resultados. Las herramientas de Nvivo permitieron a los investigadores establecer relaciones entre los resultados, las categorías y la cantidad de códigos que se destacaron en cada ítem, con el fin de identificar aquellos que tienen mayor recurrencia.

Cabe recordar que las categorías y subcategorías iniciales concernientes fueron:

\section{Tabla 1}

Categorías y subcategorías iniciales

\section{Categoría}

1. Identidad como maestros

2. Apropiación Conceptual

\section{Subcategorías}

Deber ser

Cualidades

Defectos

Metalenguaje

Lenguaje técnico sin apropiación

Apropiación sin lenguaje técnico

$\mathrm{Y}$, como categorías emergentes, se tuvo una relacionada con la reflexión en torno al sistema educativo y tres sobre el proceso de escritura:

\section{Tabla 2}

Categorías y subcategorías emergentes

3. Apreciaciones sobre el sistema Imaginarios sobre la educación infantil educativo

Apreciaciones sobre la clase:

-Negativas sobre la clase 


\begin{tabular}{cc}
\hline & Positivas sobre la clase \\
- Sobre la profesora & \\
Comentarios sobre el sistema educativo & - Positivos \\
& - Negativos \\
1. Estructura Textual & Subcategorías \\
2. Modo del discurso & Título \\
& Introducción \\
Desarrollo \\
Cierre \\
Argumentativo \\
Descriptivo \\
Expositivo \\
Reflexivo \\
Mixto \\
Cohesión \\
Coherencia \\
Organización de ideas \\
Ortografía \\
\hline
\end{tabular}

1. Los puntos de Ilegada. Finalmente, los autores formularon conclusiones, recomendaciones y propuestas. Las mismas que se exponen a continuación.

\section{Resultados}

Como se ha descrito, la estrategia Mis ideas en tres minutos nació por el interés de promover en los maestros en formación una actitud crítica y reflexiva sobre su práctica docente, sin embargo, sirvió también para identificar algunos fenómenos relacionados con la escritura que merecen atención. El siguiente gráfico de comparación por número de códigos muestra evidencias al respecto. 


\section{Figura 1}

Reporte de Nvivo sobre cantidad de códigos por categorías de análisis

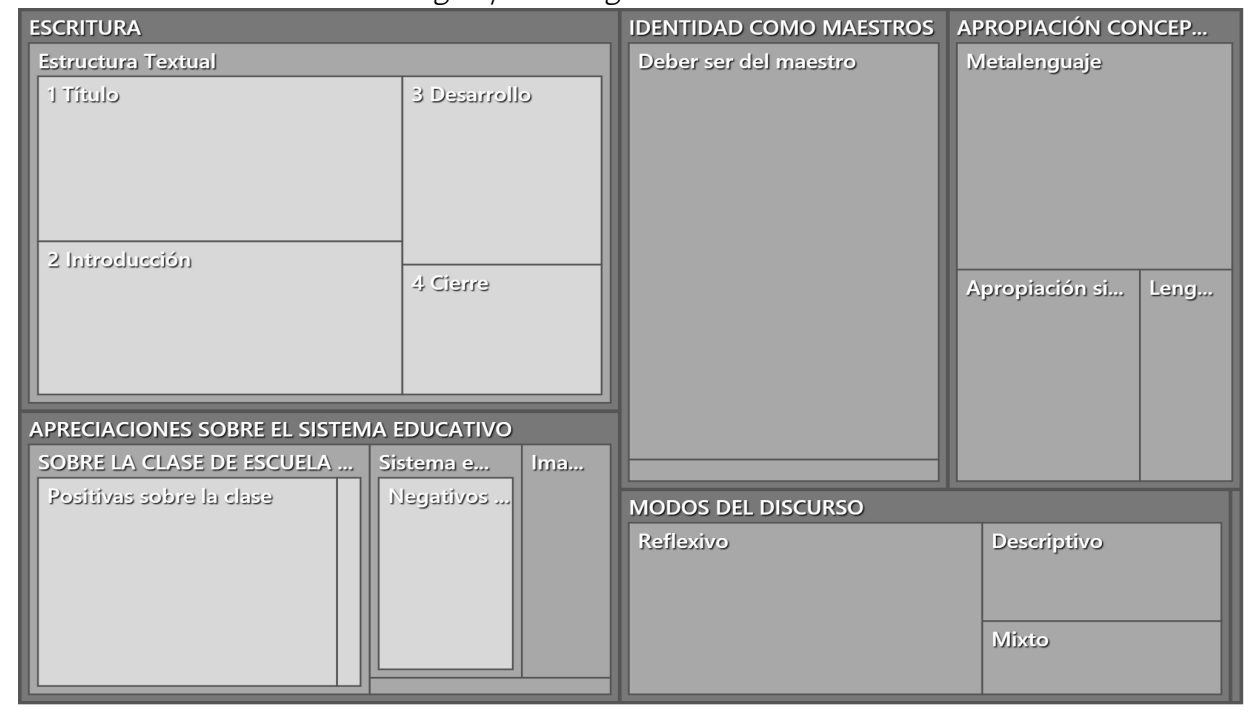

Cabe anotar que el programa Nvivo grafica los resultados de cada categoría con cuadros que ilustran el número de coincidencias encontrados en los textos. Por ejemplo, la categoría Identidad como maestros es la de mayor recurrencia; en contraste con la concerniente a Modos del discurso que es aquella que tiene menor número de referencias. Esta es una manera visual de reconocer los hallazgos del ejercicio.

A continuación, se describen los resultados alcanzados desde las categorías tanto iniciales como emergentes.

\section{Categorías iniciales}

Sobre la identidad docente de los futuros maestros, se destaca la subcategoría "deber ser del maestro", pues es la de mayor recurrencia en todo el ejercicio. Este es el principal argumento para demostrar la valía de la técnica One minute paper para fomentar procesos críticos de los estudiantes que estén en relación directa con sus aprendizajes, elemento que toma una mayor trascendencia si se considera la utilidad del ejercicio en la promoción del aspecto reflexivo de carácter permanente y espontáneo en los futuros maestros del país. Así se puede apreciar en los siguientes fragmentos: "Comprendí que, como maestra, debo tener en cuenta los pequeños detalles que hacen la diferencia, que soy garante de derechos y debo proteger la vida, teniendo en cuenta lo que necesitan los niños y lo que puedo aportar" (Estudiante 1, 2019); "Algo muy importante en el deber ser del maestro es el aprender a escuchar, lo que implica mucho más que oír" (Estudiante 4).

Tenemos, como maestros, el deber de ser buenos observadores, saber escuchar, ser cariñosos, ser respetuosos, ya que por medio de estas cosas podemos llegar a saber en qué puede el niño estar fallando y poderlo ayudar, es importante crear un vínculo, conexión, para que así le generemos una mejor confianza al niño de expresarnos lo que siente y quiere decir (Estudiante 2, 2019). 
El ser docente y tener que enfrentarnos a una población de niños tan pequeños requiere que tengamos alerta todos nuestros cinco sentidos y estar pendientes de todo lo que puede suceder en el aula de clase, nuestro tiempo, atención, cuidados, actividades, amor, afecto, escucha (Estudiante 6, 2019).

Nosotros, como docentes, somos unas de las personas que más influyen en la vida de nuestros estudiantes, por lo tanto, es muy importante la relación que tenemos con los padres de familia, para que el proceso de aprendizaje sea enriquecido con las costumbres y culturas que tienen todos los estudiantes (Estudiante 7, 2019).

También se destacó la categoría de apropiación conceptual, de la cual derivaron las subcategorías: uso de metalenguaje, comprensión de la teoría sin uso de lenguaje técnico y uso del lenguaje técnico sin apropiación. Así, se pudo reconocer que varios estudiantes usaban los conceptos de manera correcta y contextualizada; mientras que otros simplemente los repetían de forma indiscriminada. También fue posible inferir, en ciertas intervenciones, que los maestros en formación comprendían los conceptos a pesar de que no usaban los términos exactos. Aquí, algunos ejemplos:

\section{Tabla 3}

Ejemplos de fragmentos de texto que muestran evidencia de apropiación conceptual

Uso de metalenguaje La pedagogía de la crianza permite profundizar el cómo, como maestros de escuela maternal, podemos sistematizar de manera explícita lo que los niños y las niñas de cero a dos años aprenden de manera espontánea en sus hogares junto con el acompañamiento de sus pares tal y como lo mencionaba Rosa Violante en su artículo. (Estudiante 9, 2019)

Comprensión de la teoría sin uso de lenguaje técnico
Como maestros, podemos sembrar un granito de amor, confianza, etc., en cada niño para su futuro y su buen desarrollo, debemos estar muy bien articulados con sus familias, esto facilita poder entender los contextos y situaciones que se presenten y así sea más fácil aportar nuestra ayuda. (Estudiante 4, 2019)

Descubrí que, gracias al escuchar a los niños, se está generando y fortaleciendo la vincularidad, un factor muy importante. (Estudiante 8, 2019)
Uso del lenguaje técnico sin apropiación
Otra muestra de la fuerza del aspecto reflexivo del ejercicio es fácilmente apreciable en el gráfico de frecuencia de palabras (Figura 2). Allí, se encuentran las palabras "niño" y "niños" como 
las más relevantes. También llama la atención que el vocablo "cómo" tiene un papel protagónico en los discursos, al igual que expresiones que muestran el sentido de pertenencia de los futuros licenciados con su profesión, por ejemplo: maestro y docente, acompañado por conjugaciones en primera persona como "debemos" y "podemos".

\section{Figura 2}

Gráfico de frecuencia de palabras

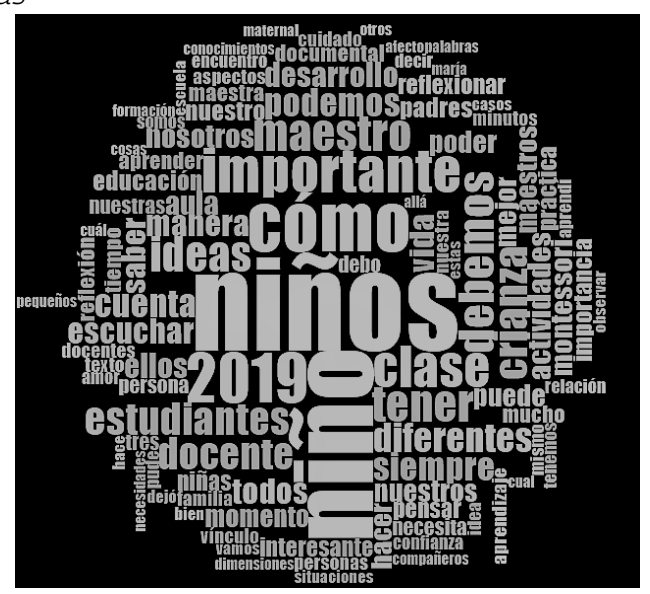

Nota: gráfico elaborado en el software Nvivo con los títulos de los 150 textos elaborados por los estudiantes.

A partir del ejercicio de codificación, se construyó un diagrama de análisis de conglomerados, lo que permite identificar qué categorías están interrelacionadas y cuáles no: 


\section{Figura 3}

Gráfico de análisis de conglomerados

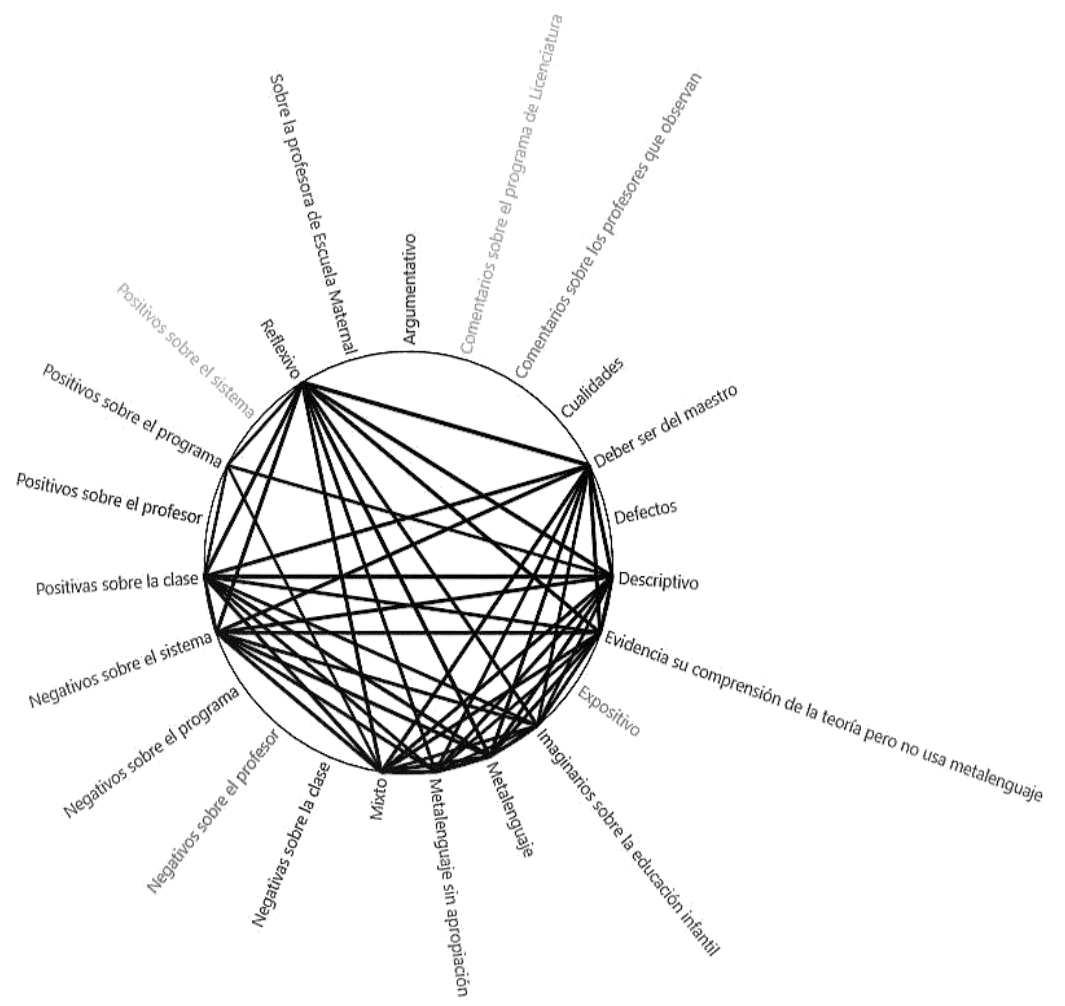

Nota: elaborado en el software Nvivo integrando todos las categorías de análisis y sus respectivos códigos.

Gracias a este diagrama, se reconocen varios elementos importantes. En primera instancia, es evidente la relación entre la actitud reflexiva de los estudiantes, los aspectos positivos de la clase, los aspectos negativos del sistema educativo, las subcategorías sobre el uso de metalenguaje y las intervenciones sobre el deber ser del maestro.

Desde los textos con orientación reflexiva elaborados por los estudiantes, se evidenció cómo los mismos exponen los aprendizajes alcanzados y también, de manera muy genuina, sus apreciaciones sobre la clase, lo que se convirtió para la docente en retroalimentación de su práctica pedagógica. Al finalizar cada clase, la asesora podía reconocer los logros a nivel conceptual que alcanzaban sus estudiantes y así mismo identificar qué había resultado significativo para ellos de lo orientado en cada sesión de seminario pedagógico, no solo por el gusto que representara, sino por el impacto en su formación. Contar con estos reflejos de manera inmediata permitió valorar las actividades, tiempos y recursos planteados en clase. También, y de manera emergente, resultó para la docente motivo de orgullo leerse en los textos de los estudiantes como una maestra digna de ser tomada como ejemplo.

En el gráfico, es evidente el carácter reflexivo de los textos, sin embargo, en los mismos, algunos aspectos no fueron tenidos en cuenta; ejemplo de ello es la reflexión sobre sus cualidades, lo cual cuestiona sobre por qué si los licenciados en formación hacen tanto hincapié en el deber ser 
como maestros, no reconocen las cualidades con las que cuentan. Quizá esto se deba a que, en su proceso de formación, se ubican aún desde el rol de estudiantes que se están preparando para ser maestros, por ello los señalamientos frente a cómo deben ser, pero no consideran relevante escribir o reflexionar sobre sus actuales fortalezas, a pesar de que estas se evidencian en su desempeño como practicantes en las diferentes instituciones educativas.

\section{Categorías emergentes}

Sobre el sistema educativo, es innegable la relevancia de los apuntes que hicieron los estudiantes, específicamente en los aspectos positivos de la clase de Práctica Escuela Maternal, comentarios negativos sobre el sistema educativo en Colombia e imaginarios de la educación inicial en el país. La siguiente tabla presenta algunos ejemplos:

\section{Tabla 4}

Ejemplos de fragmentos de texto que muestran apreciaciones de los estudiantes sobre el sistema educativo Aspectos positivos de la En el encuentro pedagógico de hoy, tuve la oportunidad de clase de Práctica de Escuela Maternal reconocer el trabajo que he realizado en mis prácticas pedagógicas, así mismo pude reflexionar acerca del crecimiento personal y profesional que he tenido desde el primer día hasta hoy. (Estudiante 1, 2019)

Comentarios negativos sobre el sistema educativo en Colombia

Imaginarios sobre la educación infantil
La educación, por su preocupación de cumplir con el currículum, ha olvidado de dónde viene el niño y quiénes lo rodean, o sea, la familia, además de todas las acciones que se realizan en la crianza de los más pequeños. (Estudiante 6, 2019)

Mi labor como maestra en educación infantil, pues va más allá de "cambiar pañales" como se dice en ciertos lugares; por ello, debo prepararme de la mejor forma para brindar una formación adecuada y significativa a los niños. (Estudiante 15, 2019)

Sobre el proceso de escritura, se pudo observar que, en términos de estructura textual, casi todos los estudiantes asignaron títulos a sus escritos. Así mismo, se encontró que las producciones incluían una idea introductoria o de contextualización; sin embargo, la presencia de un desarrollo argumental de los planteamientos y de una proposición de cierre fue menos evidente en los escritos.

Otra categoría de aspecto formal de la escritura refiere los modos del discurso, mostrando que la función reflexiva del lenguaje fue la de mayor relevancia en el ejercicio, seguida por la descriptiva y la mixta, es decir, una combinación de descripción y reflexión. De otra parte, las 
anotaciones sobre redacción no aparecieron en el reporte de Nvivo, cuestión que se debe al escaso mejoramiento de la expresión escrita que se obtuvo en el desarrollo de los textos tal como se evidencia en la Figura 3, en la que se muestran los resultados de la evaluación del componente escrito que fue denominado Redacción y que incluye elementos como: cohesión, coherencia, organización de ideas y ortografía. En aras de asignar un juicio sobre la presencia de estos componentes en los escritos, se creó la siguiente escala: 2 puntos = cumplido; 1 punto = parcialmente cumplido; y 0 puntos = sin evidencia. A lo largo de la implementación del ejercicio Mis ideas en tres minutos, no se evidencia evolución en este aspecto.

\section{Figura 3}

Desempeño de los estudiantes en el componente Redacción

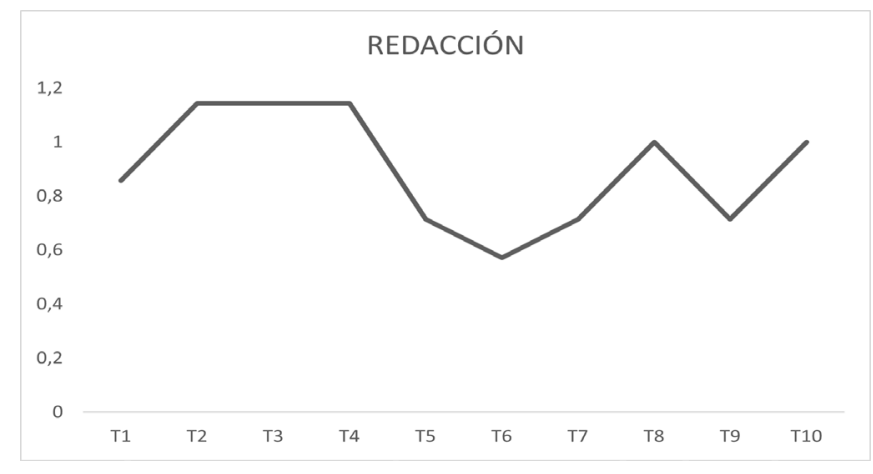

En un escenario ideal, se esperaría que, así como las producciones de los estudiantes se enriquecieron en el componente crítico y reflexivo a medida que progresaba la actividad, también lo hicieran en el escritural; no obstante, la Figura 3 muestra que lastimosamente Mis ideas en tres minutos no ayudó para que los estudiantes escribieran documentos dotados con mayor cohesión, coherencia, organización de ideas y mejor ortografía. Como se observa, el punto de llegada (1.2) es similar al punto de inicio (0.8). Una de las posibles causas de esto es la poca relevancia que en algunas ocasiones los estudiantes dan a la retroalimentación realizada por la docente; por ejemplo, varios estudiantes repetían los errores ortográficos y sintácticos en los textos a pesar de que la profesora ya se los había explicado en retroalimentaciones previas.

De igual modo, el análisis permitió reconocer algunas de las dificultades más preponderantes en términos de expresión escrita. En primer lugar, la organización clara de ideas, lo que denota que los estudiantes no tienen el hábito de la planificación textual. También pudo apreciarse que los aprendices carecen de estrategias argumentativas para sustentar fuertemente sus afirmaciones. En tercera instancia, fue evidente que los participantes no acostumbran a hacer una revisión final de lo que escriben antes de considerar que han terminado sus trabajos. 


\section{Conclusiones}

Lo primero que puede afirmarse es que Mis ideas en tres minutos es un ejercicio que favorece la reflexión permanente de la práctica y el constante cuestionamiento de la labor docente. A propósito, es importante mencionar que los estudiantes se acostumbraron a la actividad y hacían la veeduría de modo que, en cada clase, se tuviera el espacio para llevarla a cabo. Incluso, en varias ocasiones, solicitaron más tiempo para registrar sus reflexiones.

De igual forma, los estudiantes asumieron el ejercicio con gran aprecio a causa de la dedicación de la profesora del curso. Esto se pudo percibir en el hecho de evaluar los textos, revisarlos semana a semana, darles la retroalimentación y asignar a cada uno su propio portafolio, lo que generó un sentido de pertenencia de los participantes con la actividad. Adicionalmente, fue fundamental para el buen desarrollo de la experiencia que la evaluación fuera cualitativa sin incidencia alguna en su calificación y que, desde el principio, se haya aclarado que el propósito de la actividad era propiciar un espacio para que cada uno expresara de manera genuina sus pensamientos, reflexiones y sentimientos en torno a su formación como maestros.

Otra conclusión del trabajo es que Mis ideas en tres minutos permitió que la maestra investigadora hiciera un seguimiento a la dinámica del curso, pues el hecho de leer al término de cada clase todos los textos elaborados por los estudiantes le permitía tener el panorama general de lo que pasaba en cada sesión, reconocer tendencias frente a temáticas o situaciones aludidas, vacíos conceptuales, hechos impactantes y valoraciones sobre lo realizado y, en consecuencia, realizar los ajustes pertinentes. Sin embargo, solo hasta que se leyó toda la producción de cada estudiante de manera continua fue posible ver situaciones que antes no; por ejemplo, la Estudiante 4 estaba estresada con el estudio de caso que debían realizar ese semestre, pues de manera recurrente hacía comentarios en sus textos sobre la incertidumbre que tal tarea le suponía; mientras que, para la Estudiante 7, esa misma tarea resultaba un ejercicio motivante. Otra situación identificada se relaciona con las habilidades para la escritura, las cuales no evolucionaron significativamente.

Sobre la identidad docente, se observó que, en efecto, es un proceso de construcción dinámico que, como sugieren Beijaard et al. (2004), se hace parte de la dimensión interpersonal y evoluciona con el tiempo. Los textos reflejan cómo, con el pasar de las semanas, los estudiantes se lanzaban cada vez más a realizar comentarios sobre ese deber ser del maestro que se espera en la educación infantil, posicionándose como ese docente ideal que quieren ser; no obstante, todavía no reconocen las cualidades que ya han venido construyendo durante su proceso formativo.

En definitiva, Mis ideas en tres minutos fue una oportunidad para que los maestros en formación pudieran expresar sus opiniones de manera libre, sin temores a reprimendas o malas calificaciones; tampoco esperando algún tipo de premio, más que la satisfacción del ejercicio realizado y los comentarios que puntualmente daba la docente. Se puede afirmar, entonces, que, gracias a la constancia, compromiso y propósito claro con el que se desarrolló el ejercicio, este se consolidó como hábito y mostró a los estudiantes una forma efectiva de reflexionar sobre las dinámicas que se dan en las aulas de clase. 


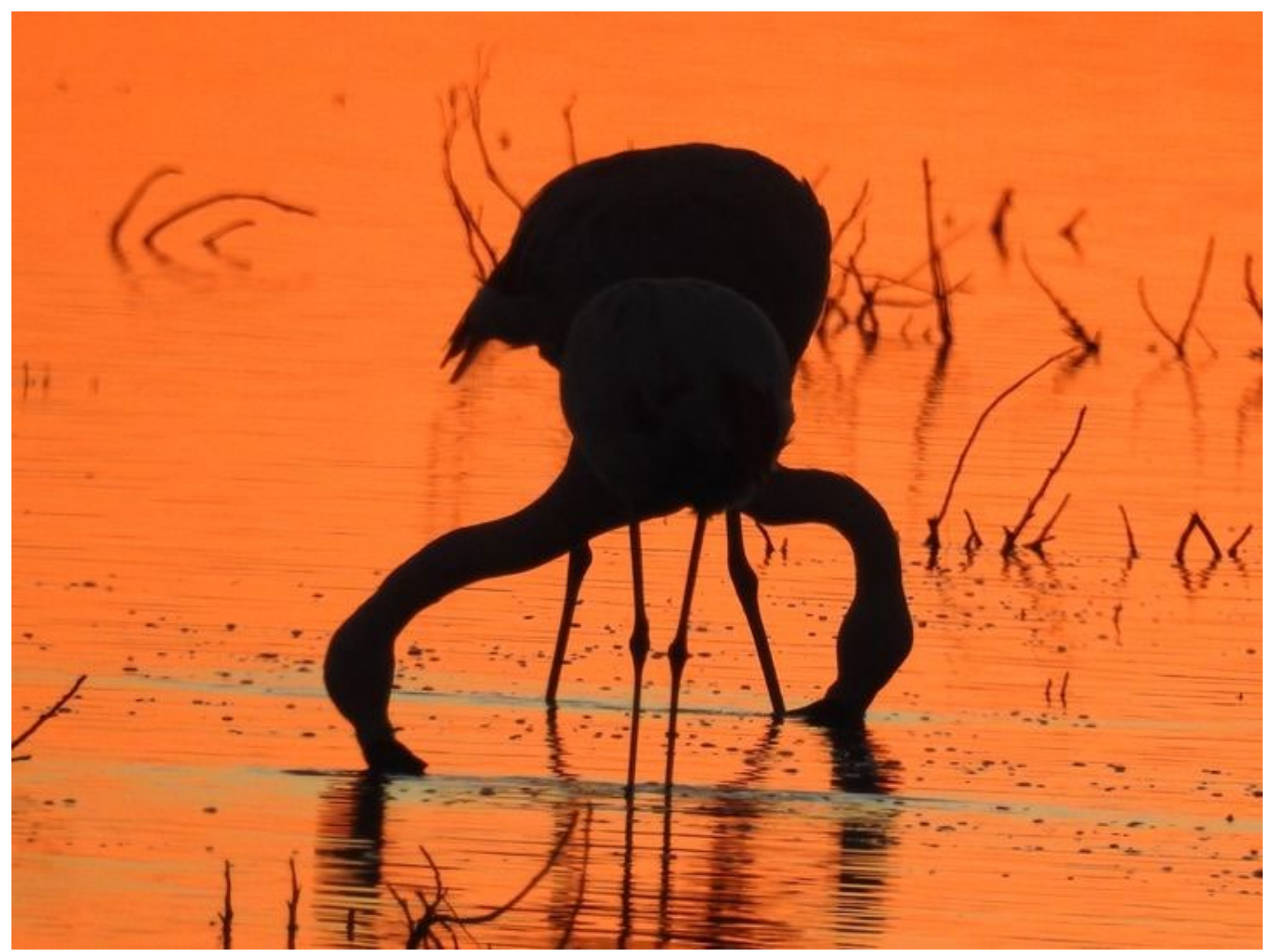

Sin título, fotografía. Andrea Talone

\section{Bibliografía}

Beijaard, D., Meijer, P. y Verloop, N. (2004). Reconsidering research on teachers professional identity. Teaching and Teacher Education, 20, 107-128.

Bocanegra, E. (2007). Las prácticas de crianza entre la Colonia y la Independencia de Colombia: los discursos que las enuncian y las hacen visibles. Revista Latinoamericana Ciencias Sociales, Niñez y Juventud, 5(1), 1-22. Cantón, I. y Tardif, M. (2018). Identidad profesional docente. Narcea.

Carlino, P. (2004). El proceso de escritura académica: cuatro dificultades de la enseñanza universitaria. Educere, Revista Venezolana de Educación, 8(26), 321-327. https://www.aacademica.org/paula.carlino/104.pdf Cross, K. P. y Angelo, T. A. (1998). Classroom assessment techniques: A handbook for faculty. National Center for Research to Improve Postsecondary Teaching and Learning.

Gairín-Sallán, J., Díaz-Vicario, A., del Arco Bravo, I. y Flores Alarcia, Ó. (2019). Efecto e impacto de las prácticas curriculares de los grados de educación infantil y primaria: la perspectiva de estudiantes, tutores y coordinadores. Educación XX1, 22(2), 17-43. www.https://doi.org/10.5944/educXX1.21311

González de Requena, J. (2019). Una historia conceptual de los metalenguajes. Revista humanidades, 9(1). Universidad de Costa Rica. https://www.redalyc.org/jatsRepo/4980/498061642005/html/index.html 
ICFES. (2019). Informe Nacional de Resultados Saber Pro 2016-2018. Subdirección de Análisis y Divulgación. https://www.icfes.gov.co/documents/20143/1711776/Informe\%20nacional\%20saber\%20pro\%202016\%20\%202018.pdf

Jara, 0. (2018). La sistematización de experiencias: práctica y teoría para otros mundos posibles. Centro Internacional de Educación y Desarrollo Humano-CINDE.

Licenciatura en Educación Infantil. (2020). Licenciatura en Educación Infantil-Profesional-Presencial. https://www.unab.edu.co/programas/licenciatura-educaci\%C3\%B3n-infantil-profesional-presencial.

MEN. (2014). La práctica pedagógica como escenario de aprendizaje. MEN.

MEN. (2017). Bases curriculares para la educación inicial y preescolar. MEN.

Programa de Licenciatura en Educación Infantil-UNAB. (2018). Reglamento de Prácticas Pedagógicas. UNAB.

Schön, D. (1992). La formación de profesionales reflexivos. Hacia un nuevo Diseño de la Enseñanza y el aprendizaje en las profesiones. Paidós.

Tobio, C., Agulló, M., Gómez, M. y Martín, M. (2010). El cuidado de las personas, un reto para el siglo XXI. Fundación "La Caixa".

UNAB. (2020). Consolidado de resultados Saber pro UNAB 2016-2019. Dirección de docencia.

UNESCO. (1992). Educación para todos, finalidad y contexto. Conferencia Mundial sobre Educación para todos. Organización de las Naciones Unidas.

Vivel-Bua, M., Fernández López, S. y Lado-Sestayo, R. (2015). Innovación docente con One Minute Paper, ¿afecta el rendimiento académico? Revista electrónica de investigación educativa, 17(2), 48-61. Recuperado el 07 de octubre de 2020 de http://www.scielo.org.mx/scielo.php?script=sci_arttext\&pid=S160740412015000200004\&Ing=es\&t|ng=es. 